\title{
Employees' witnessed presence in changing organisations
}

\author{
John Mendy
}

\begin{abstract}
In recent years, governments, businesses and other organisations have increasingly been forced to attempt to survive by reorganising themselves fundamen- tally. Although this happens at present on a large scale, it is not unprecedented. In fact, most organisations have had to change their working practises at some time for some reason-for example, when the competition catches up or when technology threatens to make production obsolete. The usual strategy is to fire part of the staff and to redis- tribute tasks. This tends to put a heavy burden on staff. They have to search for other jobs or attempt re-skilling. Those remaining may face substantial changes in their relations to their managers, who will require changes ... 'or else'. The study reported in the paper focuses on people's reactions to this approach. Some employees accept and leave. Others become aware of an opportunity to damage their company. Others again see a way to resist and change the way the managers behave and thereby turn around the company and maintain or increase the number of jobs. The study focuses on two questions. The first is how one should model or describe the behaviour of people in the third category, i.e. those wishing to contribute. It does not seem useful to follow one's first hunch, i.e. to search for quan- tifiable patterns. People's behaviour will always be con- textualised as a reaction to what managers do. This suggests the use of qualitative methods. The second ques- tion is whether the concept of presence may clarify the behaviour and identify ways for employees to contribute positively to changes in their organisations. An obstacle to answering the second question is that qualitative methods focus on people's experiences in some context, and on the emergence of their behaviour, but do not necessarily lead to suggestions on how to behave with what effect. It is attempted to answer the second question and thereby the first question with the aim of identifying what people may do who are not immediately fired or made redundant when fundamental organisational changes are introduced. Data have been collected from four companies that decided to initiate such changes in 2003-2004.
\end{abstract}

Keywords Presence $\cdot$ Actor-presence $\cdot$ Increasing presence $\cdot$ Witnessing

\section{Introduction}

The study reported in the paper concerns the experiences of employees whose company aims to change its working conditions in some fundamental way. The need for doing so may stem from changes in the market, in technology or otherwise. Companies may wish to respond by reducing their activities or expanding them. This means that employees may no longer be able to do the work they once were contracted for. They leave the company or face changes in the organisational structure. The focus of this paper is on the way employees react to such changes, in particular when still employed, whether temporarily or in the long term. Of particular interest is the possibility that they attempt to change the way their managers behave, for example to resist uncalled for impositions or to increase their contribution to the advancement of their company.

The interest in this aspect of planned change is relatively unusual. Most studies on organisational change concentrate on the overall behaviour of companies and their strategies and tend to neglect people's experiences. For example, Flood (1990: 16) describes how he 'liberated' (his actual word) 6,000 of a company's 10,000 employees. No word is spent on the impact of his actions on the lives of 
the employees (Goffman 1959). He also does not refer to any attempt employees may make (in particular those not immediately leaving) to contribute to the company's con- tinuation. It seems safe, however, to assume that there were such contributions.

The first question that arises is how to describe what was left out in such studies-as a useful step in an attempt to understand what employees experience when their organ- isations change and to make it possible to use this under- standing in similar organisations. It requires naming the experiences. Comte (1854) saw such naming as the aim of social scientists: to find a name (or model or theory) to some named (domain, modelled or data). He emphasised, however, that something else is necessary. It should be possible to use the name to recognise the named-a cri- terion that has become standard in most what is defined as research (Popper 1980; Rorty 1991). This raises the second question: what type of name will allow this to happen. The two types of names (one as an answer to the first question, one to the second) need not be the same. The first often is referred to as data, i.e. as the names of individual behav- iour, whilst the second is considered theorising or model- ling. Without being able to name both ways, i.e. twofold, it will not be possible to profit from the experiences of employees and, for example, recognise whether what happens is as intended. An example of this kind of naming is a map-as a name of names (data), i.e. as a way to recognise new events as related to old events. It has been said that such a map (or name) is 'not the territory' (Kotarbinski 1990) and that there is more to a map than what it describes. It helps individuals to recognise territo- ries they have not visited yet-via the systematised use of the experiences of others.

In many areas of study, names are part of a language (Quine 1970). Sometimes a single language seems to suf- fice-for example the language of variables in the natural sciences (where names mainly refer to variables). The social sciences appear to differ in this respect. It has become necessary to identify more than one language-as detailed in the next section. In the rest of the paper, an attempt is made to identify a name (of named data) for the experiences focused on. It is proposed to use the notion of presence. This notion will be linked to qualitative data, i.e. answers to questions about people's experiences. This requires solving a special problem. Such data do not allow for a 'twofold' name. It proved necessary to use an inter- mediate name, in this case that of a story (linking a number of stages), as a way to help recognise individual strategies to 'increase presence'.

\section{Modelling}

Many early studies of organisations tend to use the lan- guage of variables in the form of so-called trivial input- output models ('trivial' is their official name, not the author's; von Foerster 1993). Over time, the models used have become more complex and have been expanded to include parameters (context-dependent variables) and state-spaces (Chernoff 1953). A second development saw the use of control models; models that are used to effec- tively achieve or maintain some aim or objective (Beer

1966). A third group contains models of organised col- lectives, i.e. models that identify how a number of elements (not necessarily with human-like behaviour) are or become linked (Kaufman and Rousseauw 1990). A fourth type refers to coordination systems or groups of elements the aim of which is to perform some activity (Hollan 1992). The four types (and the studies in which the models have been used) identify an inability: each new type of model has been introduced to deal with the problem of not being able to achieve research quality (in the sense of Comte 1854, and others; see above). In other words, none of the models appears to provide the 'twofold' name being looked for-leaving the naming of the data as their best contri- bution. The main difficulty seems to be that the models depend on observation, whilst organisations strongly depend on objectives, plans, visions and the like.

A more promising model surfaced recently (Kaufmann and Tödtling 2001; Kaufmann et al. 2009). It is referred to as the fifth model. A number of (human and possibly non- human) actors are assumed to co-operate to achieve some action, even when the individuals' past and future intentions and objectives differ. This implies a normative model or a model for. Such models include 
values like a will- ingness to collaborate and an indication of where to go or what to achieve. They also depend on the people using the model-when being modelled. This means that they introduce the possibility of self-naming: the name given to the collective can be used by individual members and what members contribute ('names') can be recognised as required by the collective. One may differ about how to refer to this type of quality-self-naming or self-repre- senting, or more popularly, self-organisation. The term self-naming will be used in the rest of the paper.

The transition to the models of the fifth level from those of the first four levels seems important in the history of studying organisational change. It implies something of a watershed. The first four types, for example the input- output models, are intended to 'represent' patterns in the behaviour of individuals, i.e. name them and make them recognisable. Representational models of this kind tend to be used to describe what may be defined as passive behaviour, i.e. behaviour that is not proactive (does not deal with the future) and not interactive (does not react to the naming). Such models identify what input to provide if one wishes to realise a preferred output, and hence may be used effectively in the control paradigm (if the models are of sufficient quality, of course). The models of the fifth level have been developed to deal with the alternative, that is, situations where behaviour is proactive and/or interac- tive. A different structure is needed, therefore: changes in behaviour have to be part of the models. This means the models are not representational and not part of the control paradigm. If they are to be used, such use must be part of the behaviour being modelled (as data) as well as serve as guide (name) to that behaviour. The obvious example is some collective where the model 'of' its achievements is also the model 'for' the behaviour of the individuals that collectively achieve that behaviour. This type of modelling appears to be quite powerful. It is analogous to members getting together to mirror the activities of those who developed the fifth model, and hence attempting to become a collective researcher themselves. To achieve research quality in the sense of Comte (1854), the collective has to develop its own language as a way to co-ordinate the behaviour of its members. It can be expected that there will be more than one language and that they differ from the language of variables.

Some of the aspects of the process of developing collectives (as part of the use of the fifth model) seem worth emphasising. One is that the more the collectives distinguish themselves from their environment, the more relations between members become mutual. Members no longer will be able to act as single individuals as this would imply that they aim to achieve personal objectives other than those of the collective and hence will not achieve the required type of quality. For the same reason, one cannot expect that some members aim to dominate others, i.e. impose the objective of domination over the collective objective. A direct corollary is that members have to respect and trust each other (or behave such that respect can be inferred). They have to respond to each other (i.e. act upon their observations of each other), make themselves addressable, insist on the transparency of each other's objectives (or if one wishes, each other's subject position) and make themselves, their objectives as well as their feelings of commitment mutually identifi- able. These activities may be facilitated by the develop- ment of and adherence to rules, as an expression of members taking responsibility to accept and help maintain each other's contributions. Adherence to the rules may be delegated to a referee, i.e. a special kind of observer. Additionally, remaining a member must be attractive- and hence collaboration must be assumed to help achieve something that each member prefers in some sense (not necessarily the same), including that it does not detract from a member's well being, psychologically as well as physically.

Following the literature, these characteristics (as names or data concerning a certain type of collective) seem to be effectively summarised (and named) using the concept of 'presence' (Nevejan and Brazier 2011; Nevejan 2009). In terms of such 'presence', members 'witness' each other, thereby contributing to the 'dynamics of negotiating trust and truth' (Nevejan 2009: 59). What seems especially relevant is the possibility of using the name 'increasing presence' rather than only 'presence') when employees start to strengthen their interaction with their managers and colleagues to help determine the direction of their com- pany. This name should capture important aspects of what happens when employees start to engage their managers to create researcher- 
ship, possibly as a way to resist being fired, but mainly to stop the latter (or more generally, those representing their company) from treating them as persons without presence (even whilst

physically present). Such treatment has been referred to as instrumental (Habermas 1987).

As the fifth type of model focuses on actions (intentional behaviour) and is instantiated by multiagent collectives or systems, its combination with the concept of presence will be referred to as the 'actor-presence model' (or APM). The APM differs from the notion of presence as used by Nevejan (2009) and others in that it includes the notion of research quality (as that which collectives strive for), and thus should allow for the identification (or naming) of 'increasing presence', as defined-as a consequence of increasing the coherence and the level of 'self-naming' of collectives. The concept will be used to study the behav- iour of employees in four companies that can be argued to have been under sufficient stress to engage in full or complete re-organisation. It is expected that the results not only will demonstrate that some employees move towards

'increasing presence' as defined, but also that they help others to do so. This kind of result would highlight an interesting and valuable aspect of what employees can do in organisationally difficult times. It also would add evi- dence in support of the applicability of the concept of 'presence' as a way to theorise about some aspects of social change and as a way to model such change in terms of the (fifth) 'Actor-Presence Model'.

\section{Research path}

It was decided to explore whether employees can be said to aim to 'increase their presence' when their companies decide to re-organise and what will help others do the same. To collect the necessary experiences - the data (or names) in the context of the present study -, a series of interviews was organised. As it was expected that such data would depend on the interviewees' aims and commitments, a qualitative form of analysis was deemed appropriate. It was used to group the responses and order the groups into a sequence of steps as part of a story, the story of 'increasing presence'. Interviewees (51 in total) were selected based on their organisations having recently gone through some restructuring on a regional and/or national scale. Four sets of employees (from four companies; see next section) were categorised as either managers or employees. This classification emphasised differences in roles and in experiences of organisational change. The companies present a relative wide range of activities.

\section{The companies}

The four companies operate in Lincolnshire and Notting- hamshire, UK. All intended to modify the scale of their operations and, thereby alter and enhance employees' contributions, by expanding into new areas to deal with market pressures, in particular the areas of Leicestershire, Cambridgeshire and Bedfordshire.

\subsection{Longhurst housing association (referred to as Longhurst)}

The Longhurst Group is the parent of a group of companies that provide housing services (Longhurst Housing Associ- ation). The houses may be rented for a contracted time or sold. The staff comprises nearly a thousand Human Resource specialists, administrative/clerical staff, electri- cians, cleaners, builders, plumbers, architects and so on. The interviews were held in Boston where the head office is located.

\subsection{Eden supported housing (referred to as Eden)}

The company provides care and housing services to approximately a hundred and fifty people with learning and other disabilities as well as with health problems in Lin- colnshire, Nottinghamshire 
and parts of Yorkshire. Since its inception, the provision of care services has continued to grow. The company aims to expand by facilitating people living independently. This requires recruiting and training staff with the required skills. Most of the inter- views were held in Newark.

\subsection{Laurens patisserie (referred to as Laurens)}

This company is the UK's largest cake manufacturer. It produces cream cakes for supermarkets such as Tesco, Morrisons, Asda and Sainsburys. It also caters for smaller orders for private functions. The company needs to expand and integrate into its new parent company, the Bakkavor Group, with headquarters in Iceland. This Group specia- lises in freshly prepared foods with an approximate annual turnover of $£ 1.6$ billion and over forty factories in China and Europe. The interviews were held in Newark.

\subsection{Prospects (referred to as Prospects)}

The company provides educational support, career advice and counselling services to students in secondary schools. It provides support to community and youth projects, recruitment fairs and school inspection in the public, pri- vate and voluntary sectors. The annual turnover has grown since its start in 1995 to £42 million, partly by the acqui- sition of new businesses and contracts in the East Mid- lands, for example Connexions. Prospects' Headquarters are in London, where the interviews were held.

\section{Analysis of the responses}

Interviewees were asked about their experiences during the process of change, for example experiences concerning the behaviour of their managers, the way each company had been organised and had tried to change, the aims of the change, the difficulties, etc. Responses were taken to refer to the way each interviewee named certain experiences (and excluded others) and hence, that they would not necessarily have the same meaning even when they looked the same. The responses were grouped to indicate how individuals referred to each other, i.e. made themselves responsible for each other as part of a presumed collective, and attempted to 'increase their presence'. Another reason for the grouping was to identify whether the responses would suggest a systematic way to 'increase one's presence' (and show the 'twofold' nature required in research). To this end, the responses were categorised as a sequence of steps or stages, which might help employees to achieve a strong 'presence' in their company. Each stage is taken to identify how individuals may operate on the results of previous stages as a way to move their companies and themselves in a pre-ferred direction (that of competence and sustainable growth) and achieve a strong 'presence' themselves. Other responses that seemed to indicate a different story, e.g. a descent into despair, are not reported here. Some references to similar findings in the literature are added.

\section{Stages in employees' experiences}

The results of analysing the interview responses are pre- sented via six headings or stages, in combination with 

quotes from the interviewees and references to similar findings in the literature. Together they should clarify what is expected or recommended to happen at the stage. They may be interpreted as constraints or rules to channel employees' behaviour. The stages construct a 'meta-story' from what the responses appear to tell, that of individuals

'increasing presence'. The first stage identifies the starting point of the story. Its previous stage is of course what may be called the 'normal' stage of the company's develop- ment-before it attempts to change or has any reason to change.

\subsection{Experiencing the need to change}

The first stage refers to managers and employees becoming aware of the challenges their organisations are facing. Both groups emphasise the difference between previous times-when values such as openness, collegi- ality, good communication and teamwork dominated-and present times when these values become less recog- nisable. Relevant quotes suggest that the 'normal' state had been heavenly:

'I think we [at Longhurst] have a very tolerant culture...' (4.46.2).

'[Eden] staff have always been made to feel valued' (5.47.1).

'..[Eden] members of staff are also encouraged to work from home depending on their circumstances. Training and development are actively encouraged and promotion is encouraged internally' (2.3.2).

Before the organisations attempted large-scale changes, the need for managers to treat employees with respect appears to have been negligible. In all four companies, when it was decided to change, there were two first reactions, both at the same time. The first was to increase variety-make plans, extend development into the future, look for new resources, etc. The second was to restrict the variety that employees could contribute via extra discipline and 'hard' measures, for example:

'It [power] is not abused but at times, hard decisions have to be made [at Eden]' (9.9.2).

'Some managers [at Laurens] do not have the right

ideas for [the] job and as such do not have a good relationship with staff. They get easily annoyed and start shouting on staff if things go wrong' (3.17.2)

Effort and time are being spent (by managers and, in some cases, by trade unions) to create what Hö pfl (1992) refers to as the 'right' or desired employee. Individual differences and skills are downplayed. Notions of choice and involve- ment start to be replaced by increasing emphasis on who is

'boss' (Handy 1995: 7, 39; Rocha 1999).

\subsection{Drawing the boundaries}

In the second stage, managers introduce additional discipline and stricter measures to control the work force. Employees amend their working practises, to assimilate the changes and explore ways to resist them. This includes employees not turning up for work and in general an informalisation of procedures:

'Staff [at Eden] attempts to make work arrangements less strict and to erase job boundaries' (1.2.5).

'Jobs [at Longhurst] are given out verbally to a team of members within the ICT Department. This is more of an informal kind of arrangement as opposed to laid-down policies and procedures' (1.2.1).

At Eden and Longhurst, staff start to devise their own ways of conducting daily operations. Prospects' staff at Lincoln change working practises at odds with their London counterparts. These changes are 
the opposite of what managers aim to introduce. The latter gradually become aware of the resistance to their new 'ways of doing things' (Schein 1985: 17; Lundy and Cowling 1996). At Laurens staff cite 'big [employee] turnover figures' (3.16.2) and managers 'trudging on' (3.17.2) as if to suggest they are

'not bothered' (3.18.2) with longer-term development. Individual departments start to develop alternative com- munication channels to deal with the challenges, which in some cases, result in additional acts of resistances, such as

'culture jamming' (Eco 1976; Dery 1993) and taking extended breaks.

Managers respond by openly, consciously manifesting what employees consider improper emotional outbursts (Fineman 2000; Wilson 1999). They do not help in an already tense environment and promote de-motivation of employees who: 'don't want to work here [at Laurens]' (3.16.2). They may be due to excessive pressure from senior managers (Fineman 2000, 2003: 218; Bauman and Vecchi 2004). This replicates emotional spillage on employees further down the hierarchy, further de-motivating already repressed employees.

The immigration of relatively large groups of predominantly Eastern Europeans at Laurens introduces a heightened awareness of (sub)-cultural and group values at Laurens, as well as tendencies to conserve these.

\subsection{Reinforcing change}

The third stage refers to the development of new strategies: managers starting to emphasise higher, managerial values as well as reinforce mechanised forms of managing. Management methods become increasingly strict so employees feel reduced to mechanisms. Alternative forms of communication develop to bypass the managers.

Managerial methods include having staff 'constantly' (2.31.3 and 1.5.1) report to line managers and supervisors about whether they are meeting deadlines, production and delivery targets, and are improving performance (Nicholls

2004) (6.31.3):

'Others undergo a lot of pressure and job stress because of the daily targets' (3.18.2).

The 'hard' measures (Rittel and Weber 1973; Peters and Waterman 1982; Tjosvold 2007) increasingly structure behaviour, rather than concepts like welfare and care (Höpfl 1992; Wilson 1999; Fineman 2000). Longhurst employees have to discuss performance targets through staff development initiatives (SDIs) as demands rise and quality regulations become scrutinised. Personal dignity and humaneness become under-valued.

\subsection{Fuzziness over roles and personal life}

Managers start to identify with their (harsh) strategies, and blur the boundary between the personal and organisational. Employees develop new individual roles that differ from their formal ones and start to increase the variety in their behaviour. They re-discover values like dignity and selfrespect. Managers become de-motivated.

People are forced not to go beyond their roles; they are

prevented from contributing in the direction of increased competence (Hammer and Champy 1995: 13, 15, 16; Peters and Waterman 1982: 320). Employees feel like Production, Cleaning and Dispatch machines. This forces new individual identities to emerge, each attempting to go beyond the treatment (Clarke et al. 1994).

Non-managers express a sense of 'losing' something but also of becoming aware of a feeling of 'value'. Managers formulate strategies as if the procedures in their depart- ments can be described as input-output models. Employees start to increase variety to create conditions under which such models do not fit. They emphasise the importance of a second point of view next to that of managers: 
'People [at Laurens] would say, we used to do this and that...' (4.46.2).

'...junior support staff [at Eden]...confide to us trainers' (3.22.2).

\subsection{Transferring initiative}

The fifth stage sees employees starting to communicate with fellow victims of the managerial strategies and to initiate support groups (e.g. developing sub-cultures in the various departments). Managers start to withdraw from their strategies to ensure their personal survival, and, in that sense, to initiate forms of resistance of their own (mirroring non-managers), which they do by an increasing emphasis on their power:

'Managers and shareholders have the power here [at

Laurens] because they own the land' (9.9.3).

Employees are not expected to become more competent in performing collective tasks (Reid and Barrington

2004, 2007; Jakupec and Garrick 2000; Harrison 2005; Simmonds 2003). Their existing skills are downplayed. Management responses to problem-solving do not, in employees' views, address the longer-term difficulties (e.g. providing sustainable education and counselling services to young adults).

\subsection{Developing actor-presence}

Non-managers increasingly behave like independent actors in their company, fully aware of their contributions. They create new collectives in which they support the develop- ment of others, with new forms of communication. They contribute to their company's competence. These activities demonstrate an increasing ability of employees to modify or move the boundary between the different systems they participate in: those of home, of work, of relaxation, of friendships and other activities.

Managers attempt to re-establish communication-but their communication with non-managers remains formal and hierarchical. Computer technology helps to make communication less formal, however, as e-mails allow for faster flows of information:

'We [at Laurens] work as a team that is increasingly self-supportive' (1.31.15).

'I think more communication [at Laurens] is needed because of the increasing turnover figures' (4.18.1)

'Management [at Prospects] need to be talking to staff more informally' (5.52.2).

Relationships at Prospects result in tensions that are new to the organisation and the community. On the one hand, there is an increasing awareness that non-managers no longer consider managers as particularly competent. On the other, managers continue to attempt to de-personalise employees:

'Jobs are allocated based on responsibilities [at Eden]' (1.10.1)

\section{Increasing presence}

Developments in the four companies appear to show that at least some employees (non-managers) develop a positive attitude towards changes when companies face new challenges. They appear to become more innovative than their managers. They prove able to develop strong positions in the new organisation. Their 'weapon' (if one wishes to use the war metaphor-or otherwise, that from which they derive their ability to act effectively) consists of activities that may be fittingly named 'increase in presence'. It consists of the development of collectives that self- 
organise into forms of interaction where differences in status (e.g. managers versus non-managers) become less important and where collective results are like those of research, i.e. stable against threats (external ones such as market challenges and internal ones such as the use of input-output models by managers). This type of develop- ment introduces a form of modelling (actor-presence modelling) that differs from the usual form of observational representation. It proved viable in that the responses of people engaged in fundamental reorganisations could be ordered such that the development is represented in a coherent sequence of steps or stages, as well as in a 'hero-like' story that recommends how one may support and strengthen the modelling. The notion of 'presence' proved essential in the process. It made it possible to name the development of certain individuals as an 'increase in presence'.

\section{Conclusion}

It has been argued that profiting from certain forms of employee experiences requires a form of modelling that differs substantially from the representational form. It includes the modelled into the modelling process and pro- vides them with a direct link to practise. They do not only represent them. A core aspect of this form of modelling is that it requires people to engage with others in research-like activities and thereby become witnesses to their co-development. It is strongly linked to the notion of 'presence' as it can be shown to support improvements in trust, but also in physical and mental health. Referred to as actor-presence modelling, it makes it possible to describe when people are able to resist certain treatments as well as why and how, for example to support their companies or damage and even destroy them. The methods supporting such modelling include forms of qualitative analysis, but also the devel- opment of sequences or stories that serve as descriptions as well as criteria. The paper contains an example of their application. It is a story that refers to the way employees coordinate their activities (manage their contributions given the company's aims) and to the results (the realisation of the aims) -when driving developments towards more cooper- ative and respectful forms of behaviour.

The Actor-Presence Model was introduced as the result of two contributions. The first concerned the difficulties that representational modelling has to face when modelling organisations and other forms of embedded behaviour. These difficulties required the introduction of increasingly complex models that were categorised in terms of four types. It was concluded that a fifth or relational type of model would be advisable, a fundamentally different type of model. It would allow for the naming of a large amount of variation, i.e. variation that might be increased by the use of the model to achieve organisational change (e.g. Fiske et al. 2002). The second contribution concerned certain aspects of this fifth type of model; in particular its emphasis on the experiences of individuals interacting with other individuals to achieve some task, whilst attempting to satisfy the research criterion (introduced via the work of Comte (1854), but clarified in the work of many other authors on research). It was argued to be necessary, but also shown to be useful, to emphasise the concept of 'presence', as a way to describe subjective experiences as part of an objectively observable entity, i.e. a collective striving to change itself. Further work is needed ...

\section{References}

Bauman Z, Vecchi B (2004) Identity conversations with Benedetto Vecchi. Polity Press, Cambridge Beer A (1966) Development control and design quality. Sheffield Centre for Environmental Research, Sheffield Chernoff H (1953) Locally optimal designs for estimating parameters.Annals of Mathematical Statistics, Stanford University,

Stanford Clarke H, Chandler J, Barry J (1994) Organisation and identities: texts and readings in organisation behaviour. Chapman Hall, London

Comte A (1854) Système de Politique Positive. Appleton and Co, New York 
Dery M (1993) Culture jamming, hacking, slashing and sniping in the empire of signs. Addison-Wesley, New York Eco U (1976) A theory of semiotics. Indiana University Press, Bloomington

Fineman S (2000) Emotions in organisations. Sage, London Fineman S (2003) Understanding emotions at work. Sage, London Fiske ST, Cuddy AJC, Glick P, Xu J (2002) A model of (often mixed) stereotype content: competence and warmth respectively follow. J Pers Soc Psychol 82(Part 6):878-902. doi:10.1037/0022-3514.82.6878

Flood RL (1990) Liberating systems theory. Plenum Press, NY Goffman E (1959) The presentation of self in everyday life. Penguin, Harmondsworth

Habermas $J$ (1987) The theory of communicative action, volume 2, system and life-world: a critique of functionalist reason. Beacon Press, Boston

Hammer M, Champy J (1995) Reengineering the corporation: a manifesto for business revolution. Nicholas Brealey Publishing, New York

Handy C (1995) Beyond certainty: the changing world of organisa- tions. Chatham Mackays of Chatham plc, Kent Harrison R (2005) Learning and development. CIPD, London

Hollan JH (1992) Adaptation in natural and artificial systems. TheMIT Press, Cambridge

Höpfl H (1992) Values and valuations: the conflicts between culture change and job cuts. In: Clarke H, Chandler J, Barry J Needle D (2000) Organisation and management: a critical text. Thompson Learning, London

Jakupec V, Garrick J (2000) Flexible learning, human resource and organisational development: putting theory to work. Routledge, London

Kaufman L, Rousseauw PJ (1990) Finding groups in data: an introduction to cluster analysis. Wiley, New York

Kaufmann A, Tö dtling F (2001) Science-industry interaction in the process of innovation: the importance of boundary-crossing between systems. Res Policy 30(5):791-804. doi:10.1016/s0048-7333(00)118-9

Kaufmann A, Lehner P, Tödtling F (2009) Do different types of innovation rely on specific kinds of knowledge interactions? Technovation, Elsevier 29(1): 59-71. doi: 10.1016/jtechnovation.2008.05-002

Kotarbinski T (1990) Kotarbinski: logic, semantics and ontology. In: Wolenski J (ed). Kluwer, Dordrecht

Lundy O, Cowling A (1996) Strategic human resource management. Routledge, London

Nevejan C (2009) Witnessed presence and the YUTPA framework. Sprouts PsychNology J 7(1):59-76. ISSN1535-

6078

Nevejan C, Brazier F (2011) Witnessed presence in merging realities in healthcare environments. Stud Comput Intell 326:201-227. doi:10.1007/978-3-642-16095-0_11

Nicholls A (2004) Social entrepreneurship. Financial times handbook of management, 3rd edn, pp 636-643

Peters T, Waterman R (1982) In search of excellence. Harper and Row, New York

Popper KR (1980) The logic of scientific discovery. Unwin Hyman, New York

Quine WW (1970) Philosophy of logic. Prentice Hall, Englewood Cliffs

Reid MA, Barrington H (2007) Training interventions: managing employee development, 5th edn. Jaico Publishing House, Mumbai

Reid MA, Barrington H, Brown M (2004) Human resource develop- ment: beyond training interventions. CIPD, London

Rittel H, Weber M (1973) Dilemmas in a general theory of planning. Policy Sci 4:155-169

Rocha JF (1999) Continuous frequency control from 5 to $10 \mathrm{GHz}$ of an opticaloscillator, frequency and time forum 1999 IEEE and the international frequency control symposium. In: Proceedings of the joint meeting of the European, vol 2. pp 602-603. Cat.No.99CH36313

Rorty R (1991) Consequences of pragmatism. Harvester, London

Schein EH (1985) Organizational culture and leadership. Jossey-Bass, San Francisco

Simmonds D (2003) Designing and delivering training. CIPD, London

Tjosvold D (2007) Cooperative and competitive goal approach to conflict: accomplishments and challenges. App Psychol 47(3):285-313. doi:10.1111/j.1464-0597.1998.tb00025.x von Foerster H (1993) KybernEthik, Merve, Berlin

Wilson F (1999) Organisational behaviour: a critical introduction. Oxford University Press, Oxford 